\title{
Secondary tumors of the pancreas: four cases and literature review
}

\author{
Tamas Puskas, , Imre Henits, Laszlo Kovacs \\ Department of Radiology, Markusovszky Teaching Hospital, Szombathely, Hungary
}

Received: September 15, 2014

Accepted: December 29, 2014 Online Published: January 26, 2015

DOI: $10.5430 / j b g c . v 5 n 1 p 33$

URL: http://dx.doi.org/10.5430/jbgc.v5n1p33

\begin{abstract}
Four cases affected by pancreatic metastases revealed by CT and MR during the last two years are presented. In Case 1 and 2 the metastatic disease appeared more than 10 years after the primary renal neoplasm was diagnosed. In the remaining two cases - non small cell lung cancer and non-Hodgkin disease - the metastases developed shortly after the onset of the primary malignancy. A literature review is given. Metastases in the pancreas are rare. The symptoms and imaging features are variable, therefore non-invasive imaging diagnosis is difficult. To resolve this problem a thorough scrutiny of the patients' medical history, and the functional imaging methods can be helpful.
\end{abstract}

Key Words: Pancreatic metastases, Non-invasive imaging diagnosis

\section{Introduction}

In most cases, space occupying lesions developing in the pancreas are primary pancreatic tumors. Primary tumors of other organs rarely form metastases in the pancreas, and their clinical and radiological characteristics are different. Therefore some times the exact diagnosis is difficult. Over the past two years, at the Radiological Department of Markusovszky Hospital in Szombathely, pancreatic metastases originating from tumors of three other organs were diagnosed, and the authors briefly reviewed the related literature.

\section{Case presentation}

\subsection{Patient 1 (64-year old male)}

Removal of the left kidney due to hypernephroma in 1999. The patient regularly underwent chest and abdominal follow-up CT and ultrasound examinations. In 2011 the ultrasound found widening of the pancreatic body and tail. Chest and abdominal CT scans revealed multiple small subpleural nodules in both lungs, and an inhomogeneous contrast enhancing lesion in the pancreatic tail. The first diagnosis was pancreatic tumor with lung metastases. No biopsy was performed because the unsafe approach of the mass. Re-evaluation of CT examination definitively concluded pancreatic and lung metastases of the previous renal tumor. The patient receives oncological medical treatment; his condition is unchanged. On follow-up examinations lung metastases did not change in number or size, while the pancreatic metastasis grew slightly (see Figure 1).

\subsection{Patient 2 (52-year old female)}

Left-side nephrectomy due to hypernephroma in 1999. The patient attended periodical check-ups. In 2012, a lesion developed in the right thyroid lobe, which was removed. The histological examination result was renal cell carcinoma

\footnotetext{
*Correspondence: Tamas Puskas; Email: uskas.amas@freemail.hu; Address: Department of Radiology, Markusovszky Teaching Hospital, Szombathely, Hungary.
} 
metastasis. Chest and abdominal CT scans found lesions typical for a renal tumor in the pancreas and the right kidney, which were considered to be metastases of the earlier renal tumor. Both secondary tumors were verified by $\mathrm{CT}$ guided biopsies. Accordingly, the patient receives appropriate oncological medical treatment. His condition is satisfactory, he has not lost weight and he performs light work without any difficulty (see Figure 2).

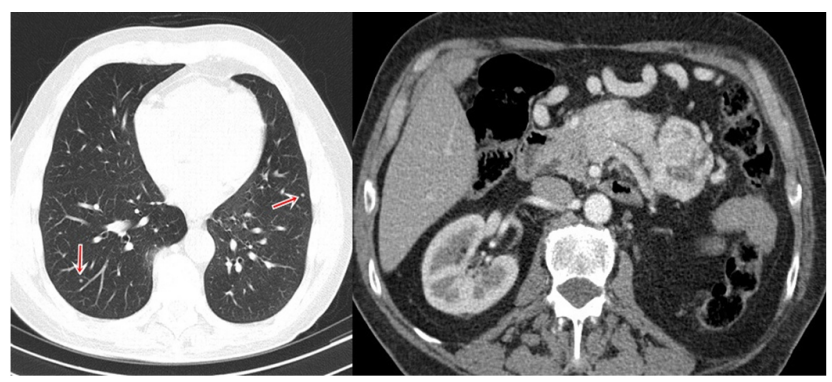

Figure 1: Left kidney removed because of tumor. In the pancreatic tail a metastatic mass, typical enhancement for renal-cell carcinoma, can be seen. There are some small subpleural nodules in both lungs (arrows).

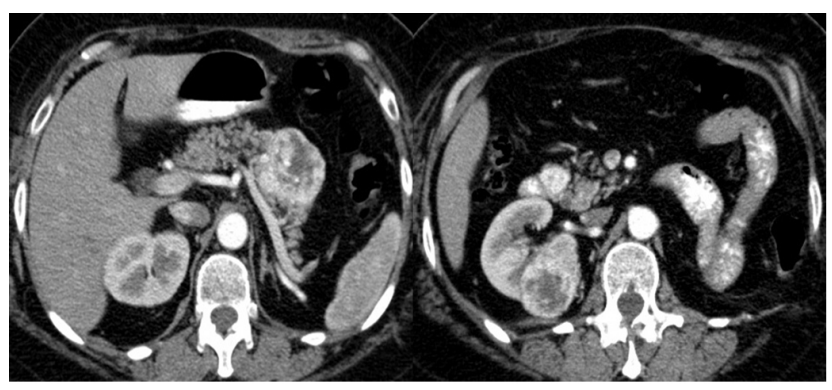

Figure 2: Left sided nephrectomy. Two lesions typical for a renal tumor, one located in the pancreatic body, the other one in the middle part of the right kidney.

\subsection{Patient 3 (57-year old male)}

Pulmonectomy and mediastinal lymph node dissection in February 2011 due to a central lung tumor and left lower lobe atelectasis. Examinations for staging performed before surgery found no abnormalities in the abdominal organs. In March 2011, the patient suffered mild pancreatitis. One month later developed jaundice, intra-, and extra-hepatic bile ducts were enlarged. After an unsuccessful ERCP procedure, the abdominal MRI and MRCP revealed two distinct lesions located in the head and body of pancreas. In addition behind the pancreas an enlarged lymph node could be seen. On diffusion-weighted sequences the lesions definitely showed restricted diffusion typical for malignancy. The diagnosis was pancreatic and lymph node metastases of the lung tumor. Icterus resolved after biliary stent implantation, however, the patient's general condition rapidly worsened, and the patient died in October. Autopsy confirmed the multi-organs metastases (see Figure 3).

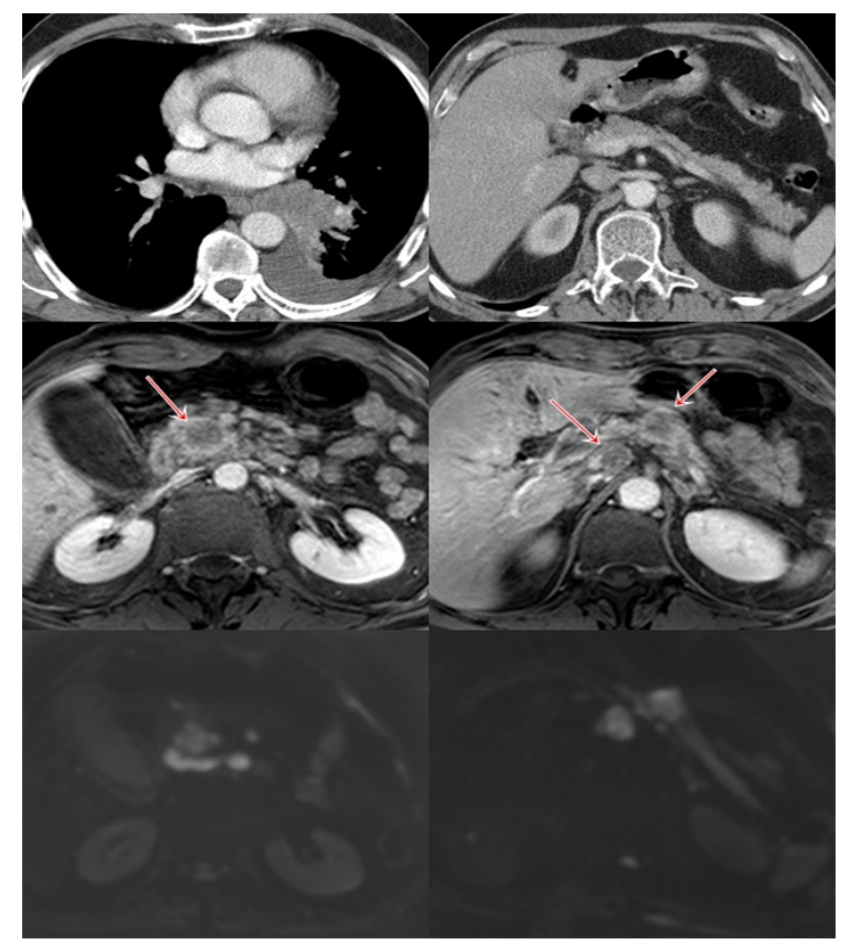

Figure 3: Upper line: Central lung tumor causing left lower lobe atelectasis. The pancreas is intact. Middle line: One month later; after contrast material administration on T1-weighted image: there are 2 circumscribed non-enhancing lesions in the pancreatic head and tail. Enlarged lymph node is located in the retroperitoneal space. Lower line: The lesions are high signal intensity on diffusion-weighted sequence with $b$ value 1,000 , a sign of malignancy.

\subsection{Patient 4 (22-year old female)}

No history of previous serious illnesses. In summer 2012, she became out of breath while playing sports and experienced night sweats. She lost $7 \mathrm{~kg}$ within a short period of time. In August, a chest CT scan found a $7.5 \mathrm{~cm} \times 6 \mathrm{~cm}$ mass in the anterior mediastinal region. Biopsy from the lesion confirmed the diagnosis of large B-cell lymphoma. At the same time, the patient also complained of non-specific abdominal pains. Abdominal CT and MRI scans demonstrated two well-circumscribed masses in the pancreatic head and body. No enlarged lymph nodes were found in the abdomen. As a result of haematological treatment the follow-up CT examination (October 2012), the mediastinal mass showed significant decrease, while the pancreatic lesions had disappeared completely (see Figure 4).

\section{Discussion}

Pancreatic metastases are rare, it's about 5\% of all pancreatic malignancies. ${ }^{[1-3]}$ They cannot be easily diagnosed, since they are partly asymptomatic, or are associated with 
symptoms similar to those caused by primary pancreatic tumors. According to Hiotis' publication, 69\% of patients were asymptomatic when metastases developed only within the pancreas. ${ }^{[4]}$ Therefore, these metastases can only be detected on follow-up examinations performed due to clinical symptoms, "accidentally" during the treatment of the primary tumor, or on regular regular follow-ups. In most of the cases, an exact diagnosis is proved on the histopathological examination after surgery or autopsy. Histologically, the image of metastases developing in other organs is very similar to that of the primary tumor, and this similarity is also valid for diagnostic imaging. Pancreatic metastases may develop in multiple locations at the same time; they are generally hypervascularized, and rarely accompanied by enlarged lymph nodes. These facts may help in differentiating them from primary pancreatic tumors. According to Adsay, when processing 973 tissue samples collected during surgery, almost one third of the 38 pancreatic metastases were diagnosed as primary tumors by previous clinical investigations. ${ }^{[5]}$ Based on results of several authors from histological examinations following surgery or autopsy, pancreatic metastases most commonly originate from kidney, lung, breast and colon cancers, or malignant melanoma and more rarely from lymphoma. ${ }^{[5-7]}$

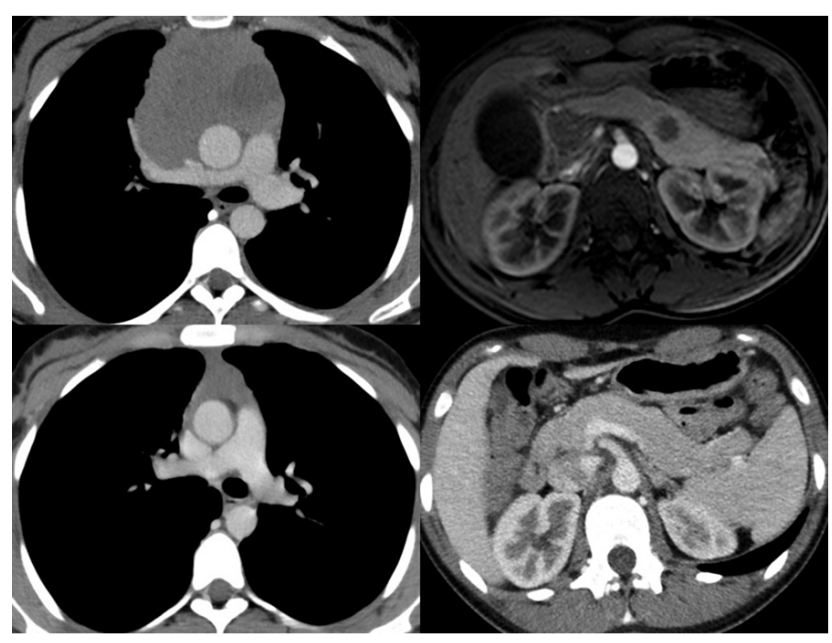

Figure 4: Upper line: A large inhomogeneous mediastinal mass; B-cell lymphoma. On contrast enhanced T1 images low signal intensity lesions are in the pancreatic head and body. Lower line: 2-month follow up examination: as a result of treatment, the size of mediastinal mass significant decreased, while the pancreatic lesions had disappeared completely.

Renal cell carcinoma. Based on the literature, renal cell carcinoma metastasizes most commonly to the pancreas. According to Sellner's publication, in $12 \%$ of renal tumors pancreatic metastases were already visible on the first diagnosis. ${ }^{[8]}$ However, the majority of metastases generally appear only 10 years after the detection and removal of the primary tumor. The development of metastases is part of

Published by Sciedu Press the systemic disease. According to literature data, left renal tumors result more often pancreatic metastases. ${ }^{[9]}$ The already developed metastases are slow-growing, and if they are resectable, the 5-year survival rate is more than $50 \%{ }^{[3]}$

In non-small cell lung cancer, pancreas metastasis is more commonly a part of multi-organ metastases. ${ }^{[5]}$ After the diagnosis of the primary disease, metastases - including those affecting the pancreas - develop rapidly, within a few months. ${ }^{[10]}$ Even if metastases are limited to the pancreas and are surgically removable, the survival period is still significantly shorter than in the case of renal cell carcinoma.

Similarly, for breast tumors, pancreatic metastases may develop first of all as a part of generalized metastatization. In larger autopsy materials, incidence was found to be $8 \% .{ }^{[6]}$ The slow-growing pancreatic metastases may appear 5-6 years after detection of the primary tumor. Combination of surgical and pharmacological treatment increases the survival period. $^{[11]}$

For colon tumors, the pancreas is affected through direct propagation of the tumor. In these cases, simultaneous removal of the colon and pancreas significantly improves life expectancy. ${ }^{[12]}$

Malignant melanoma metastasizes to visceral organs in about $20 \%$ of cases. Gutman et al. have analyzed larger material on gastrointestinal metastases of malignant melanoma, and they have found pancreatic involvement in $16 \%$ of cases. $^{[13]}$

Very few articles in the literature mention pancreatic metastases arising from lymphomas, and these were diagnosed with imaging studies and confirmed by histology. ${ }^{[14]}$ During 4,955 pathological examinations performed after autopsy, Adsay has found pancreatic metastases in 81 cases, 5 of which proved to be lymphomas. From the previously mentioned 38 pancreatic metastases 11 were lymphomas. ${ }^{[5]}$ Unlike metastases resulting from other primary tumors, lymphoma appears as a hypodense area (T1), i.e. with decreased signal intensity, showing no enhancement after contrast material administration similar to pancreatic adenocarcinoma.

\section{Conclusion}

Diagnostic difficulties related to pancreatic metastasis/metastases arise from their low incidence and the diversity of their symptoms and manifestations. Histological results can be obtained only by invasive way. Non-invasive differential diagnosis may be supported by past medical history, knowledge of previous malignant disease and typical imaging appearance of the primary tumor. The functional imaging methods in oncological staging and follow-up examinations take priority in part even today, but certainly will do so in the future. Besides PET scans, diffusionweighted MR sequences, ADC values and perfusion factors offer great possibilities in the histological diagnosis of different malignancies. 


\section{References}

[1] Brady L.W., O'Neill E.A., Farber S.H. Unusual sites of metastases. Semin. Oncol. 1977; 4: 59-64. PMid: 841351.

[2] Roland C.J., VanHeerden J.A. Nonpancreatic primary tumors with metastasis to the pancreas. Surg. Gynecol. 1989; 168: 345-347.

[3] Showalter L.S., Hager E., Yeo C., J. Metastatic disease to the pancreas and spleen. Semin. Oncol. 2008; 35: 160-171. PMid: 18396201. http://dx.doi.org/10.1053/j.seminonco 1.2007 .12 .008

[4] Hiotis S.P., Klimstra D.S., Conlon K.C. Results after pancreatic resection for metastatic lesions. Ann. Surg. Oncol. 2002; 9: 675-679. PMid: 12167582. http://dx.doi.org/10.1007/BF02574484

[5] Adsay N.V., Andea A., Basturk O., et al. Secondary tumors of the pancreas: an analysisof surgical and autopsy database and review of the literature. Wirchows. Arch. 2004; 444: 527-535.

[6] Minni F., Casadei R., Perenze B., et al. Pancreatic metastases: observation of three cases and review of the literature. Pancreatology. 2004; 4: 409-520. PMid: 15316227. http://dx.doi.org/10.11 $59 / 000080248$

[7] Volmar K.E., Jones C.K., Xie H.B. Metastases in the pancreas from nonhematologic neoplasm. Diagn. Cytopathol. 2004; 31: 216-220. PMid: 15452907. http://dx.doi.org/10.1002/dc. 20100

[8] Sellner F., Tykalsky N., DeSantis M., et al. Solitary and multiple isolated metastases of clear cell renal carcinoma to the pancreas: an indication for pancreatic surgery. Ann. Sur. Oncol. 2006; 13: 1-11. PMid: 16372157. http://dx.doi.org/10.1245/ASO.2006.03 .064

[9] Wente M.N., Kleef J., Esposito I., et al. Renal cancer cell metastasis into the pancreas. Pancreas. 2005; 30: 218-222. PMid: 15782097. http://dx.doi.org/10.1097/01.mpa.0000 153337.58105 .47

[10] Moussa A., Mitry E., Hammel P., et al. Pancreatic metastases: a multicenter study of 22 patients. Gastroenterol. Clin. Biol. 2004; 28: 872-876. http://dx.doi.org/10.1016/S0399-8320(04 ) $95151-2$

[11] Crippa S., Bonardi C., Bovo G., et al. Pancreaticoduodenectomy for pancreatic metastases from breast carcinoma. JOP. J Pancreas (Online). 2004; 5: 377-383.

[12] Berrospi F., Celis J., Ruiz E., et al. En bloc pancreaticoduodenectomy for right colon cancer invading adjacent organs. J. Surg. Oncol. 2002; 79: 194-197. PMid: 11870671. http://dx.doi.org/1 $0.1002 / j$ so. 10072

[13] Gutman H., Hess K., Kokotsakis J. Surgery for abdominal metastases of cutaneous melanoma. World. J. Surg. 2001; 25: 750-758. PMid: 11376411. http://dx.doi.org/10.1007/s00268-001 $-0027-2$

[14] Seifarth H., Schülke C., Wessling J. Non-Hodgkin lymphoma with rare metastases in the pancreas. Somatom. Sessions. 2012; 30: 5253. 